\title{
ON A REPRESENTATION OF THE MATCHING POLYTOPE VIA SEMIDEFINITE LIFTINGS
}

\author{
TAMON STEPHEN AND LEVENT TUNÇEL
}

\begin{abstract}
We consider the relaxation of the matching polytope defined by the non-negativity and degree constraints. We prove that given an undirected graph on $n$ nodes and the corresponding relaxation of the matching polytope, $\lfloor n / 2\rfloor$ iterations of the Lovász-Schrijver semidefinite lifting procedure are needed to obtain the matching polytope, in the worst case. We show that $\lfloor n / 2\rfloor$ iterations of the procedure always suffice.
\end{abstract}

1. Introduction. In integer programming, given a polyhedron in $\mathbb{R}^{d}$, we are usually interested in obtaining a description of $P_{I}:=\operatorname{conv}\left(P \cap \mathbb{Z}^{d}\right)$ in terms of linear inequalities. There has been a considerable amount of work in this area. Recently, an intriguing procedure was described by Lovász and Schrijver (1991). Their procedure takes as input a polyhedron and using the cone of symmetric positive semidefinite matrices, delivers a description of the convex hull of integer points in the original polyhedron. Each iteration of the procedure consists of one "semidefinite lifting" and one "projection." The procedure requires at most $d$ iterations.

Lovász and Schrijver illustrate their procedure on the stable set problem. One of their impressive results is that only one iteration of the procedure applied to the basic linear programming relaxation of the stable set problem (only edge inequalities and non-negativity constraints), delivers a description of a convex set for which many interesting classes of inequalities such as odd hole, odd anti-hole, and clique inequalities are valid. Moreover, any linear function can be approximately optimized over this convex set in polynomial time.

Lovász and Schrijver ask the question of how many iterations of the procedure would be needed to obtain a description of the convex hull of the incidence vectors of stable sets in a line graph. Or equivalently, how many iterations of the procedure would be needed to get the convex hull of the incidence vectors of matchings in an undirected graph, if we start the procedure with the polytope defined by the non-negativity and the degree constraints. The same problem is also mentioned in the recent survey paper of Goemans (1997).

In this paper, we prove that for a clique on $(2 n+1)$ nodes, at least $n$ iterations of the procedure are needed to obtain the underlying matching polytope. Moreover, it easily follows from a result of Lovász and Schrijver that for an undirected graph on $n$ nodes, $\lfloor n / 2\rfloor$ iterations of the procedure suffice in the worst case. Note that for the clique on ( $2 n$ +1 ) nodes, the corresponding matching polytope has dimension $d=n(2 n+1)$. Therefore, our results show that the number of iterations of the Lovász-Schrijver procedure is roughly $\sqrt{d / 2}$ on matching problems, in the worst case.

2. Matching polytope. Let $G=(V, E)$ denote an undirected graph with node set $V$ and edge set $E$. Define

Received July 22, 1997; revised May 11, 1998.

AMS 1991 subject classification. Primary: 90C10, 90C27.

OR/MS subject classification. Primary: Programming/Semidefinite, Programming/Integer.

Key words. Matching polytope, semidefinite lifting, semidefinite programming, integer programming.

1

0364-765X/99/2401/0001/\$05.00

Copyright $\odot$ 1999, Institute for Operations Research and the Management Sciences 


$$
P(G):=\left\{x \in \mathbb{R}^{E}: x(\delta(v)) \leq 1, \forall v \in V, x \geq 0\right\} .
$$

In the above, $\delta(v)$ is the set of edges in $E$ that are incident on $v$; for $S \subseteq E, x(S)$ represents $\Sigma_{j \in S} x_{j}$. For $S \subseteq V, E(S)$ refers to the set of edges in $E$ with both endpoints in $S$. Then the matching polytope associated with $G$ is

$$
P_{I}(G):=\operatorname{conv}\left\{P(G) \cap \mathbb{Z}^{E}\right\} .
$$

Edmonds (1965) proved that

$$
P_{I}(G)=\left\{x \in P(G): x(E(S)) \leq \frac{|S|-1}{2}, \text { for all } S \subseteq V \text { such that }|S| \text { is odd }\right\} .
$$

Instead of $P(G)$ and $P_{I}(G)$, we will deal with the related cones

$$
\begin{gathered}
P:=\left\{x \in \mathbb{R}^{E \cup\{0\}}: x(\delta(v)) \leq x_{0}, \forall v \in V, x \geq 0\right\}, \\
P_{I}:=\operatorname{conv}\left(P \cap \mathbb{Z}^{E \cup\{0\}}\right) .
\end{gathered}
$$

We suppressed the reference to the graph $G$, since for the most part of the paper, we will deal only with cliques on an odd number of nodes. From now on, $P(2 k+1), P_{I}(2 k$ $+1)$ refer to the cones $P$ and $P_{I}$ defined by the clique on $(2 k+1)$ nodes. We will refer to the edge set of a $(2 k+1)$ clique as $E_{2 k+1}$.

3. Lovász-Schrijver procedure. We present a brief description of one of the procedures proposed by Lovász and Schrijver. Let $K \subset \mathbb{R}^{d+1}$ denote a polyhedral cone obtained from a polyhedron in $\mathbb{R}^{d}$ via homogenization using the new variable $x_{0} . K_{I}$ denotes the cone generated by all $0-1$ vectors of $K$. The cone generated by all $0-1$ vectors $x$ $\in \mathbb{R}^{d+1}$ with $x_{0}=1$ is called $Q$.

Now, we define the lifting and projection operations. In what follows, $e_{j}$ is the $j$ th unit vector and $e$ is the vector of all ones. The sizes of $e$ and $e_{j}$ will be clear from the context. $K^{*}, Q^{*}$ are the dual cones of $K$ and $Q$ under the standard Euclidean inner-product. e.g.,

$$
K^{*}:=\left\{s \in \mathbb{R}^{d+1}: x^{T} s \geq 0, \forall x \in K\right\} .
$$

Definition 3.1. $A(d+1) \times(d+1)$ matrix, $Y$, with real entries is in $M(K, Q)$ if (i) $Y=Y^{T}$,

(ii) $Y e_{0}=\operatorname{Diag}(Y)$,

(iii) $u^{T} Y v \geq 0, \forall u \in K^{*}, v \in Q^{*}$.

Definition 3.2. $Y \in M_{+}(K, Q)$ if $Y \in M(K, Q)$ and $Y$ is positive semidefinite.

Now we define the projections of these liftings $M$ and $M_{+}$.

$$
\begin{aligned}
N(K) & :=\{\operatorname{Diag}(Y): Y \in M(K, Q)\}, \\
N_{+}(K) & :=\left\{\operatorname{Diag}(Y): Y \in M_{+}(K, Q)\right\} .
\end{aligned}
$$

We also define the iterated operators $N^{r}(K)$ and $N_{+}^{r}(K)$ as follows. $N^{0}(K):=K, N_{+}^{0}(K)$ $:=K, N^{r}(K):=N\left(N^{r-1}(K)\right)$ and $N_{+}^{r}(K):=N_{+}\left(N_{+}^{r-1}(K)\right)$ for all integers $r \geq 1$.

Lovász and Schrijver prove the following.

TheOrem 3.1. (Lovász-Schrijver (1991)). 


$$
K \supseteq N(K) \supseteq N^{2}(K) \supseteq \cdots \supseteq N^{d}(K)=K_{I},
$$

and

$$
K \supseteq N_{+}(K) \supseteq N_{+}^{2}(K) \supseteq \cdots \supseteq N_{+}^{d}(K)=K_{I} .
$$

Lovász-Schrijver also note that the condition (iii) of Definition 3.1 is equivalent to (iii') $Y e_{i} \in K$ for all $i \in\{0,1, \ldots, d\}$ and $Y\left(e_{0}-e_{i}\right) \in K$ for all $i \in\{1, \ldots, d\}$.

4. Construction of fractional points for semidefinite liftings. We turn to the matching polytope $P_{I}$ and its relaxation $P$. Consider the matching polytope of a $(2 k+1)$-clique. Let Inc $(2 k+1)$ denote the set of all pairs $\{i, j\}, i \neq j$ such that the edges $i, j$ are incident on the same node of the $(2 k+1)$-clique.

Given $v \in \mathbb{R}^{E_{2 k+1} \cup\{0\}}$, for $i \in E_{2 k+3}$ we define $w^{i}(v) \in \mathbb{R}^{E_{2 k+3} \cup\{0\}}$ as follows.

$$
w_{j}^{i}(v):= \begin{cases}1 & \text { if } j=0 \text { or } j=i \\ 0 & \text { if }\{i, j\} \in \operatorname{Inc}(2 k+3) \\ v_{j} & \text { otherwise. }\end{cases}
$$

Note that fixing any edge $i \in E_{2 k+3}$, throwing away all the edges incident on some endpoint of $i$, uniquely identifies a $(2 k+1)$ clique. Figure 1 represents the vector $w^{i}(v)$ for

$$
v:=\left(\begin{array}{c}
1 \\
(1 /(2 k)) e
\end{array}\right)
$$

When we refer to $v$, it will be clear from the context what the corresponding $k$ is.

LEMMA 4.1. Let $r$ be a nonnegative integer. If $v \in N_{+}^{r}(P(2 k+1))$ and $v_{0}=1$ then $w^{i}(v) \in N_{+}^{r}(P(2 k+3))$ for every $i \in E_{2 k+3}$.

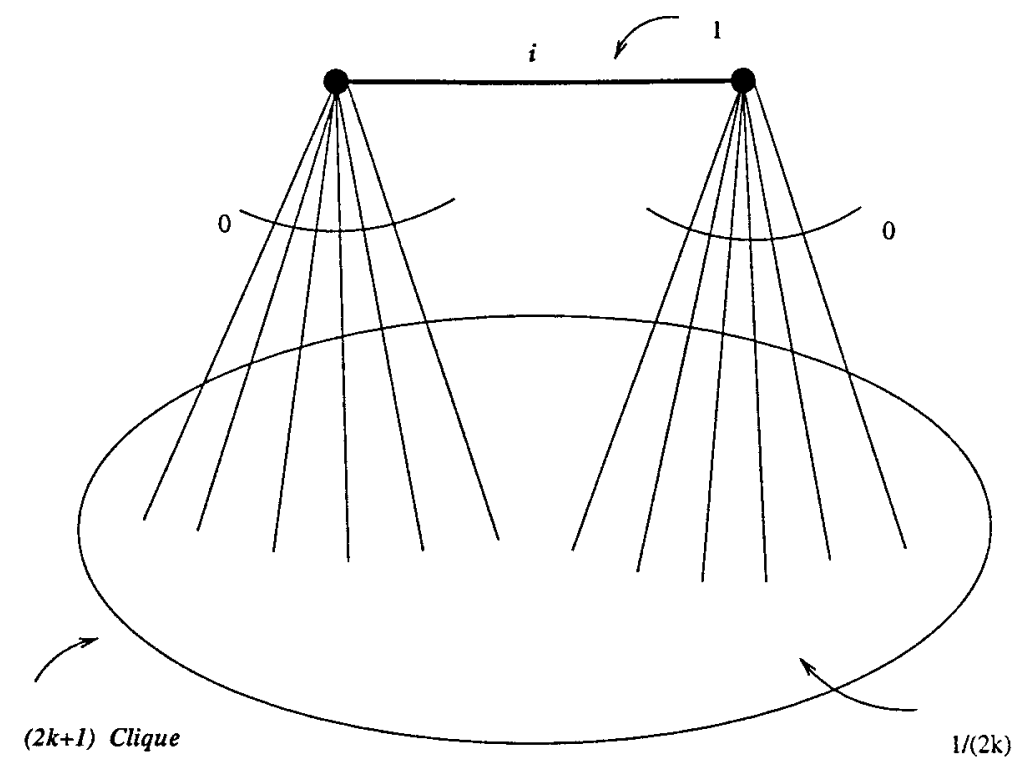

FIGURE 1. Assignment of the values on the $(2 k+3)$ clique. 
Proof. We prove the statement by induction on $r$. For $r=0$, consider the constraints defining $P(2 k+3)$. Setting $x_{i}=1$ for some $i \in E_{2 k+3}$ forces $x_{j}=0$ for all $j$ such that $\{i$, $j\} \in \operatorname{Inc}(2 k+3)$. The remaining variables have precisely the same constraints on them as those defining $P(2 k+1)$. Thus, for every $v \in P(2 k+1)$ and every $i \in E_{2 k+3}, w^{i}(v)$ $\in P(2 k+3)$. The induction hypothesis is that if $v \in N_{+}^{r}(P(2 k+1))$ then $w^{i}(v)$ $\in N_{+}^{r}(P(2 k+3))$ for every $i \in E_{2 k+3}$. Suppose $v \in N_{+}^{r+1}(P(2 k+1))$. So, there exists a symmetric, positive semidefinite real matrix, $Y$, such that $v=Y e_{0}=\operatorname{Diag}(Y), Y e_{i}$ $\in N_{+}^{r}(P(2 k+1))$ for all $i \in E_{2 k+1} \cup\{0\}, Y\left(e_{0}-e_{i}\right) \in N_{+}^{r}(P(2 k+1))$ for all $i$ $\in E_{2 k+1}$. Note that

$$
Y=\left[\begin{array}{cc}
1 & \operatorname{Diag}(\bar{Y})^{T} \\
\operatorname{Diag}(\bar{Y}) & \bar{Y}
\end{array}\right]
$$

for some $\bar{Y}$. For the $(2 k+3)$ clique we define

$$
\tilde{Y}:=\left[\begin{array}{cccc}
1 & 1 & 0 & \operatorname{Diag}(\bar{Y})^{T} \\
1 & 1 & 0 & \operatorname{Diag}(\bar{Y})^{T} \\
0 & 0 & 0 & 0 \\
\operatorname{Diag}(\bar{Y}) & \operatorname{Diag}(\bar{Y}) & 0 & \bar{Y}
\end{array}\right] .
$$

Without loss of generality, we assumed $i=1$; in the above, the block matrices of 0 s correspond to the edges $j$ in $E_{2 k+3}$ such that $\{i, j\} \in \operatorname{Inc}(2 k+3)$.

It is clear by construction that $\tilde{Y}$ is symmetric and that $w^{i}(v)=Y e_{0}=\operatorname{Diag}(Y)$. Next, we note that every principal submatrix of $\tilde{Y}$ either has zero determinant or is equal to a principal submatrix of $Y$ and hence have nonnegative determinant. Therefore, $\tilde{Y}$ is positive semidefinite.

Since $Y e_{0} \in N_{+}^{r}(P(2 k+1))$, by induction hypothesis, $\tilde{Y} e_{0}, \tilde{Y} e_{1} \in N_{+}^{r}(P(2 k+3))$. Clearly, $0 \in N_{+}^{r}(P(2 k+3))$. If $\left(Y e_{0}\right)_{j}>0$, then we have $\left(1 /\left(Y e_{0}\right)_{j}\right) Y e_{j} \in N_{+}^{r}(P(2 k$ $+1))$. Thus, by the induction hypothesis, $\left(1 /\left(Y e_{0}\right)_{j}\right) \tilde{Y} e_{j} \in N_{+}^{r}(P(2 k+3))$. If $\left(Y e_{0}\right)_{j}$ $=0$ (that is, $\left.\operatorname{Diag}(Y)_{j}=0\right)$ then $Y e_{j}=0$, since $Y$ is symmetric positive semidefinite. In this case, we again have $\tilde{Y} e_{j} \in N_{+}^{r}(P(2 k+3))$. A similar argument applies to $Y\left(e_{0}\right.$ $\left.-e_{j}\right)$ showing that $\tilde{Y}\left(e_{0}-e_{j}\right) \in N_{+}^{r}(P(2 k+3))$. We proved that $\tilde{Y} \in M_{+}\left(N_{+}^{r}(P(2 k\right.$ $+1)))$. Therefore, $w^{i}(v)=\operatorname{Diag}(\tilde{Y}) \in N_{+}^{r+1}(P(2 k+3))$.

From now on, we set

$$
v:=\left(\begin{array}{c}
1 \\
(1 /(2 k)) e
\end{array}\right) .
$$

Based on the point $v$ and Lemma 4.1, we define a matrix $Y \in \mathbb{R}^{E_{2 k+3} \cup\{0\} \times E_{2 k+3} \cup\{0\}}$ as follows.

$$
\begin{gathered}
Y e_{i}:=\frac{1}{2 k+2} w^{i}(v) \text { for } i \in E_{2 k+3}, \\
Y e_{0}:=\sum_{i \in E_{2 k+3}} \frac{1}{\left(\begin{array}{c}
2 k+3 \\
2
\end{array}\right)} w^{i}(v)=\left(\begin{array}{c}
1 \\
(1 /(2 k+2)) e
\end{array}\right)=\sum_{i \in E_{2 k+3}} \frac{2}{2 k+3} Y e_{i} .
\end{gathered}
$$

It is clear by the definition of $Y$ that it is symmetric and that $\operatorname{Diag}(Y)=Y e_{0}$; we also noted above that $Y e_{0}$ can be expressed as a nonnegative linear combination of $Y e_{i}$ s. Using this construction we prove the following lemma. 
LEMMA 4.2.

$$
\left(\begin{array}{c}
1 \\
(1 / 2 n) e
\end{array}\right) \in N_{+}^{n-1}(P(2 n+1)), \text { for all } n \geq 1 \text {. }
$$

Proof. We prove it by induction. For $n=1$, it is easy to see that $\left(\begin{array}{c}1 / 2 \\ e\end{array}\right) \in P(3)$. Then the induction hypothesis is that the statement of the lemma holds for all $n \leq k$. So, $v$ $\in N_{+}^{k-1}(P(2 k+1))$. By Lemma 4.1, $w^{i}(v) \in N_{+}^{k-1}(P(2 k+3))$ for all $i \in E_{2 k+3}$. Since $Y e_{i}$ is a positive multiple of $w^{i}(v), Y e_{i} \in N_{+}^{k-1}(P(2 k+3))$ for all $i \in E_{2 k+3}$. Since $Y e_{0}$ is a nonnegative linear combination of $Y e_{i}$ 's, $Y e_{0} \in N_{+}^{k-1}(P(2 k+3))$.

We now prove that $Y\left(e_{0}-e_{i}\right)$ also belongs to $N_{+}^{k-1}(P(2 k+3))$.

$$
\frac{2 k+2}{2 k+1}\left(Y\left(e_{0}-e_{i}\right)\right)_{j}= \begin{cases}1 & \text { if } j=0 \\ 0 & \text { if } i=j \\ \frac{1}{2 k+1} & \text { if }\{i, j\} \in \operatorname{Inc}(2 k+3) \\ \frac{2 k-1}{2 k(2 k+1)} & \text { otherwise. }\end{cases}
$$

For each $i \in E_{2 k+3}$ consider all $l \in E_{2 k+3}$ such that $\{i, l\} \in \operatorname{Inc}(2 k+3)$. There are $2(2 k+1)$ such $l$. We have

$$
\frac{2 k+2}{2 k+1} Y\left(e_{0}-e_{i}\right)=\sum_{l:\{i, l\} \in \operatorname{Inc}(2 k+3)} \frac{1}{2(2 k+1)} w^{l}(v)
$$

and thus $Y\left(e_{0}-e_{i}\right)$ is a nonnegative linear combination of elements of $N_{+}^{k-1}(P(2 k$ $+3))$. Therefore, $Y\left(e_{0}-e_{i}\right) \in N_{+}^{k-1}(P(2 k+3))$.

Next, we prove that $Y$ is positive semidefinite by identifying its eigenvalues and the corresponding eigenspaces. For convenience we scale $Y$ and work with

$$
\hat{Y}:=2 k(2 k+2) Y \text {. }
$$

The eigenvalues of $\hat{Y}$ are $\left(6 k^{2}+7 k\right),(2 k+1)$, and 0 with the ranks of corresponding eigenspaces $1, k(2 k+3)$, and $(2 k+3)$ respectively. The eigenvector corresponding to the eigenvalue $\left(6 k^{2}+7 k\right)$ is

$$
\left(\begin{array}{c}
2 k+2 \\
e
\end{array}\right)
$$

The eigenspace for the eigenvalue $(2 k+1)$ is spanned by the signed incidence vectors of even cycles where the signing forces any two consecutive edges of the cycle to have the opposite sign (we set the 0th component to 0$)$. We claim that there are at least $k(2 k$ +3 ) linearly independent such vectors. Take a simple cycle $C$ on all $(2 k+3)$ nodes. For every edge $j \in E_{2 k+3} \backslash C, C \cup\{j\}$ has one odd and one even cycle containing $j$. For each $j \in E_{2 k+3} \backslash C$, consider the signed incidence vectors of the even cycle containing edge $j$. These vectors are linearly independent (since each $j$ appears only in one such cycle) and there are 


$$
\left(\begin{array}{c}
2 k+3 \\
2
\end{array}\right)-(2 k+3)=k(2 k+3)
$$

of them. Next, we identify the eigenspace of the eigenvalue 0 . Fix a node $h_{1} \in V_{2 k+3}$. For each $h_{2} \in V_{2 k+3} \backslash\left\{h_{1}\right\}$, consider the complete bipartite graph whose bipartition is $\left\{h_{1}\right.$, $\left.h_{2}\right\}, V_{2 k+3} \backslash\left\{h_{1}, h_{2}\right\}$. Assign weight 1 to every edge incident on $h_{1}$, assign weight -1 to every edge incident on $h_{2}$ in this bipartite graph. Define the 0th entry and all the other entries to be 0 . Such a vector is in the eigenspace corresponding to the eigenvalue 0 . It is easy to check that these $(2 k+2)$ vectors are linearly independent. Finally,

$$
\left(\begin{array}{c}
-(2 k+3) / 2 \\
e
\end{array}\right)
$$

is also a vector in the eigenspace defined by the eigenvalue 0 . So, we have at least ( $2 k$ +3 ) linearly independent vectors in the eigenspace of 0 . Since the sum of the lower bounds we proved for the eigenspaces equals the order of the matrix $\hat{Y}$, we have identified all eigenvalues of $\hat{Y}$. Since each of the three eigenvalues is nonnegative, $\hat{Y}$, and hence $Y$ is positive semidefinite. We proved

$$
Y \in M_{+}\left(N_{+}^{k-1}(P(2 k+3))\right),
$$

which implies that

$$
\operatorname{Diag}(Y)=\left(\begin{array}{c}
1 \\
e /(2 k+2)
\end{array}\right) \in N_{+}^{k}(P(2 k+3)) .
$$

This completes the induction.

5. Analysis of the Lovász-Schrijver procedure for the matching problem. We summarize the consequences of the above construction in the next theorem.

THEOREM 5.1.

$$
N_{+}^{n-1}(P(2 n+1)) \supset P_{I}(2 n+1)=N_{+}^{n}(P(2 n+1)) .
$$

Proof. By Lemma 4.2,

$$
v=\left(\begin{array}{c}
1 \\
e / 2 n
\end{array}\right) \in N_{+}^{n-1}(P(2 n+1)), \quad \text { for all } n \geq 1
$$

Since

$$
\sum_{j \in E_{2 n+1}} v_{j}=\frac{2 n+1}{2}>n
$$

the maximum cardinality of any matching in the $(2 n+1)$ clique, $v \notin P_{I}(2 n+1)$. Using Theorem 3.1, we have the strict inclusion. For the equality, note that

$$
P_{I}(2 n+1) \subseteq N_{+}^{n}(P(2 n+1))
$$


again by Theorem 3.1. For the converse, we apply Lemma 1.5 of Lovász and Schrijver (1991) in an induction. We will prove that the inequality

$$
x(E(S)) \leq l x_{0},
$$

where $S \subseteq V$, has cardinality $(2 l+1)$, is valid for $N_{+}^{l}(P(2 n+1))$. It can be checked by direct computation that the statement is true for $l=1$. For $l=k+1$, note that setting any edge variable $x_{j}=1$, forces $x_{i}=0$ for all $i$ such that $\{i, j\} \in \operatorname{Inc}(2 n+1)$. The resulting inequality is

$$
x\left(E\left(S^{\prime}\right)\right) \leq k x_{0},
$$

where $S^{\prime} \subseteq S$ with $\left|S^{\prime}\right|=2 k+1$. By induction, the last inequality is valid for $N_{+}^{k}(P(2 n+1))$ for any choice of $j \in E(S)$. Therefore, by Lemma 1.5 of Lovász and Schrijver (1991),

$$
x(E(S)) \leq(k+1) x_{0},
$$

is valid for $N_{+}^{k+1}(P(2 n+1))$ for any $S \subseteq V$ such that $|S|=2 k+3$.

Note that the second part of the above proof applies to any undirected graph $G$, yielding

$$
N_{+}^{\lfloor|V| / 2\rfloor}(P(G))=P_{I}(G) .
$$

Consider the general setting of 0-1 integer programming problems. If $P$ and $P_{I}$ have dimension $d$, then to obtain $P_{I}$ from $P$, at least $\Omega(\sqrt{d})$ iterations of the Lovász-Schrijver $N_{+}$procedure is needed in the worst case. For the matching problem $O(\sqrt{d})$ iterations of the procedure suffice.

Acknowledgements. Research of the first author was supported in part by a summer research award from Faculty of Mathematics, University of Waterloo and by a research grant form NSERC of Canada. The second author's research was supported in part by research grants from NSERC of Canada.

\section{References}

Edmonds, J. 1965. Maximum matching and a polyhedron with 0,1-vertices. J. Res. Nat. Bureau of Standards-B 69B $125-130$.

Goemans, M. X. 1997. Semidefinite programming in combinatorial optimization. Math. Prog. 79 143-161.

Lovász, L., A. Schrijver 1991. Cones of matrices and set-functions and 0-1 optimization. SIAM J. Optim. 1 $166-190$.

Tamon Stephen: Department of Mathematics, University of Michigan, 2072 East Hall, 525 East University Avenue, Ann Arbor, Michigan 48109-1109; e-mail: tamon@umich.edu

Levent Tunçel: Department of Combinatorics and Optimization, Faculty of Mathematics, University of Waterloo, Waterloo, Ontario N2L 3G1, Canada; e-mail: 1tuncel@math.uwaterloo.ca 